\title{
Effect of magnetic field on terahertz generation via laser interaction with a carbon nanotube array
}

\author{
Santosh Jain ${ }^{1}$, Jetendra Parashar ${ }^{1 *}$ and Rajnish Kurchania ${ }^{2}$
}

\begin{abstract}
An amplitude-modulated laser, in the presence of a static magnetic field, interacts with an array of carbon nanotubes embedded on a metallic surface. The laser exerts a ponderomotive force on the free electrons of carbon nanotubes at twice the modulation frequency that falls in the terahertz $(\mathrm{THz})$ range. Each nanotube acts as an oscillatory electric dipole, producing $\mathrm{THz}$ radiations. The presence of magnetic field shifts the resonance condition and provides tunability. The THz radiation power increases with magnetic field strength.
\end{abstract}

Keywords: Terahertz radiation, Carbon nanotubes, Cyclotron resonance, Modulation frequency

\section{Findings \\ Background}

Over the last decade, there has been significant research activity in the area of terahertz $(\mathrm{THz})$ radiation generation and detection. The $\mathrm{THz}$ region of the electromagnetic spectrum lies in the gap between microwaves and infrared with a frequency range of 0.1 to $10 \mathrm{THz}$ and corresponding wavelength range of 30 to $3,000 \mu \mathrm{m}$. THz radiations have applications in defense, medical imaging, sensors, spectroscopy, etc. THz radiations can be generated via several mechanisms, viz. optical rectification of a short pulse laser or a surface plasma wave, photoconduction, Cherenkov radiation, etc. [1-9]. Presently, there is an increased interest in employing metallic nanostructures and carbon nanotubes to generate $\mathrm{THz}$ radiations as these structures offer the advantage of compactness with reasonable efficiency. A metallic nanostructured surface has been employed by Welsh and Wyne [10] to generate ultrafast $\mathrm{THz}$ radiation pulses via excitation of surface plasmon. Garwe et al. [11] have observed THz radiations on either side of a gold-coated nanograting $(500 \mathrm{~nm})$, employing a $785-\mathrm{mm}, 150-\mathrm{ps}, 1-\mathrm{KHz}$ Ti:sapphire laser. Studies on nanoscale periodic arrays of rectangular-shaped holes revealed band formation of $\mathrm{THz}$ nanoresonators [12]. Nemilentsau et al. [13] have investigated the application of a finite-length isolated

\footnotetext{
* Correspondence: j.p.parashar@gmail.com

'Department of Applied Physics, Samrat Ashok Technological Institute,

Vidisha, Madhya Pradesh 464001, India

Full list of author information is available at the end of the article
}

carbon nanotube as a thermal nanoscale antenna in $\mathrm{THz}$ range. Batrakev et al. [14] and Portnoi et al. [15] have given elegant reviews of terahertz processes in carbon nanotubes. Another promising scheme of $\mathrm{THz}$ radiation generation is by exploiting excellent metallic properties of carbon nanotubes [16]. Wang and Wu [17] have experimentally studied the properties of $\mathrm{THz}$ radiation pulses emitted by a metallic, large-aspect ratio carbon nanotube antenna. Ramakrishnan et al. [18] have observed generation of a sub-picosecond $\mathrm{THz}$ pulse by illuminating a graphite surface with femtosecond infrared laser pulses. Dragoman and Dragoman [19] have given the physical model and simulation results of semiconducting carbon nanotube resonant-tunneling diodes for $\mathrm{THz}$ radiation generation. Zhao et al. [19] have studied the characteristics of metallic, single-walled carbon nanotubes as $\mathrm{THz}$ antenna. Wang et al. [20] have observed antenna-like effect in an array of aligned carbon nanotubes. Dagher et al. [21] have studied amplification of radiation in metallic carbon nanotubes under the influence of a d.c. magnetic field. Parashar and Sharma [22] have studied $\mathrm{THz}$ radiation generation via optical rectification of a laser in an array of carbon nanotubes. In this work, we extend their work to study the effect of static magnetic field on $\mathrm{THz}$ radiation generation from an array of carbon nanotubes. An amplitude-modulated laser interacts with an array of cylindrical carbon nanotubes. The laser exerts a ponderomotive force on the free electrons on the nanotubes, inducing on them a current at twice the modulation 
frequency. The image charges in the metallic base constitute a supplementary array with currents out of phase by $\pi$ with respect to the primary array. These oscillating nanotube arrays act as antennas to produce $\mathrm{THz}$ radiation.

\section{Analysis}

Consider a metal surface $(x=0)$, embedded with normally mounted carbon nanotubes of radius $r_{\mathrm{c}}$, length $\ell_{\mathrm{c}}$ $\hat{x}$, and surface density $N_{\mathrm{c}}$ (per unit area), where $\ell_{\mathrm{c}}>r_{\mathrm{c}}$. The free electron density inside a nanotube is $n_{0} c . f$. Figure 1.

An amplitude-modulated laser is impinged on the nanotube array with electric and magnetic fields

$$
\begin{aligned}
\overrightarrow{\mathrm{E}}_{L} & =A_{L}(\hat{x}-i \hat{y})(1+\mu \cos [\Omega(t-z / c)]) e^{-i(\omega t-k z)}, \\
\overrightarrow{\mathrm{B}}_{L} & =\frac{c \overrightarrow{\mathrm{k}} \times \overrightarrow{\mathrm{E}}_{L}}{\omega} \\
A_{L}^{2} & =A_{0}^{2} e^{-\left(x-\uparrow_{c}\right)^{2} / r_{0}^{2}}
\end{aligned}
$$

where $k=\omega / c, \mu$ is the modulation index of laser, and $\Omega$ is in terahertz range.

There also exists a static magnetic field given by

$$
\overrightarrow{\mathrm{B}}_{s}=B_{0} \hat{z}
$$

Under the influence of electric field, the electrons of nanotubes are displaced by a distance $\Delta$, and the displacement is governed by the equation of motion

$$
m \frac{d^{2} \vec{\Delta}}{d t^{2}}=-e \overrightarrow{\mathrm{E}}_{L}-m \frac{\omega_{p}^{2} \Delta}{2 \varepsilon_{L}} \hat{y}-e \frac{\partial \vec{\Delta}}{\partial t} \times \overrightarrow{\mathrm{B}}_{s} .
$$

Here, $\omega_{\mathrm{p}}=\left(n_{\mathrm{o}} e^{2} / m \varepsilon_{0}\right)^{1 / 2}$ is the plasma frequency of electrons in a nanotube, $-e$ and $m$ are the charge and effective mass of the electron, $\varepsilon_{0}$ is the free space permittivity, and $\varepsilon_{\mathrm{L}}$ is the relative permittivity of the lattice.

The $x$ and $y$ components of the equation of motion can be written as

$$
\omega^{2} \Delta_{x}+i \omega \omega_{c} \Delta_{y}=(e / m) E_{\mathrm{Lx}}
$$

and

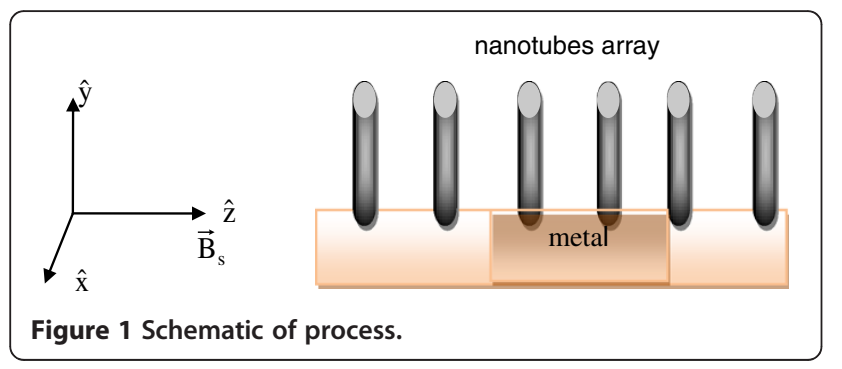

$$
\left(\omega^{2}-\omega_{\mathrm{pe}}^{\prime} 2 / 2\right) \Delta_{y}-i \omega \omega_{c} \Delta_{x}=(e / m) E_{\mathrm{Ly}}
$$

where

$$
\omega_{p}^{\prime}=\omega_{p} / \sqrt{\varepsilon_{L}}
$$

Here, it is to be mentioned that the restoring force due to the displacement of the electron cloud of nanotubes is finite for the field perpendicular to the nanotube axis and zero for the field along the axis.

On simplifying Equations 5 and 6, we obtain the $x$ and $y$ components for the displacement of the electron cloud as

$$
\Delta_{x}=\frac{e}{m}\left[\frac{E_{\mathrm{Ly}}}{\omega^{2}}-\frac{i\left(\omega / \omega_{c}\right) E_{\mathrm{Ly}}-\left(\omega_{c} / \omega\right) E_{\mathrm{Lx}}}{\omega^{2}-\omega_{\mathrm{pe}}^{/ 2} / 2-\omega_{c}^{2}}\right]
$$

and

$$
\Delta_{y}=\frac{e}{m}\left[\frac{E_{\mathrm{Ly}}+i\left(\omega_{c} / \omega\right) E_{\mathrm{Lx}}}{\omega^{2}-\omega_{\mathrm{pe}}^{/ 2} / 2-\omega_{c}^{2}}\right]
$$

respectively.

The electron velocity can be written as

$$
\overrightarrow{\mathrm{v}}=-i \omega\left(\Delta_{x} \hat{x}+\Delta_{y} \hat{y}\right)
$$

The laser exerts a ponderomotive force on electrons at the modulation frequency

$$
\begin{aligned}
\overrightarrow{\mathrm{F}}_{p}=- & \frac{e}{2 c} \overrightarrow{\mathrm{v}} \times \overrightarrow{\mathrm{B}}_{L}^{*} \\
& \cong-\hat{z} \frac{\mathrm{e}^{2} \mathrm{~A}_{\mathrm{L}}^{2} \mathrm{ki}}{2 \mathrm{~m} \omega^{2}} \frac{\omega \omega_{\mathrm{c}}}{\omega^{2}-\omega_{\mathrm{pe}}^{/ 2}-\omega_{\mathrm{c}}^{2}} \mathrm{e}^{-2 i \Omega(\mathrm{t}-\mathrm{z} / \mathrm{c})} .
\end{aligned}
$$

The oscillatory velocity of electrons due to the ponderomotive force following Equation 5 can be written as

$$
\overrightarrow{\mathrm{v}}_{2 \Omega}=\frac{i \overrightarrow{\mathrm{F}}_{p} 2 \Omega}{m\left(4 \Omega^{2}-\omega_{p}^{\prime 2} / 2\right)} .
$$

The current density at $\Omega$ can be written as

$$
\overrightarrow{\mathrm{J}}_{2 \Omega}=-n_{0} e \overrightarrow{\mathrm{v}}_{2 \Omega}=-\frac{2 n_{0} e \Omega i \overrightarrow{\mathrm{F}}_{p}}{m\left(4 \Omega^{2}-\omega_{p}^{\prime 2} / 2\right)}
$$

$\overrightarrow{\mathrm{J}}_{2 \Omega}$ is finite over the cross section $\pi r_{\mathrm{c}}^{2}$ of a nanotube and zero over the area $a^{2}=1 / N$ (where $a$ is the separation between the nanotubes). Thus, the average terahertz current density due to the array is 


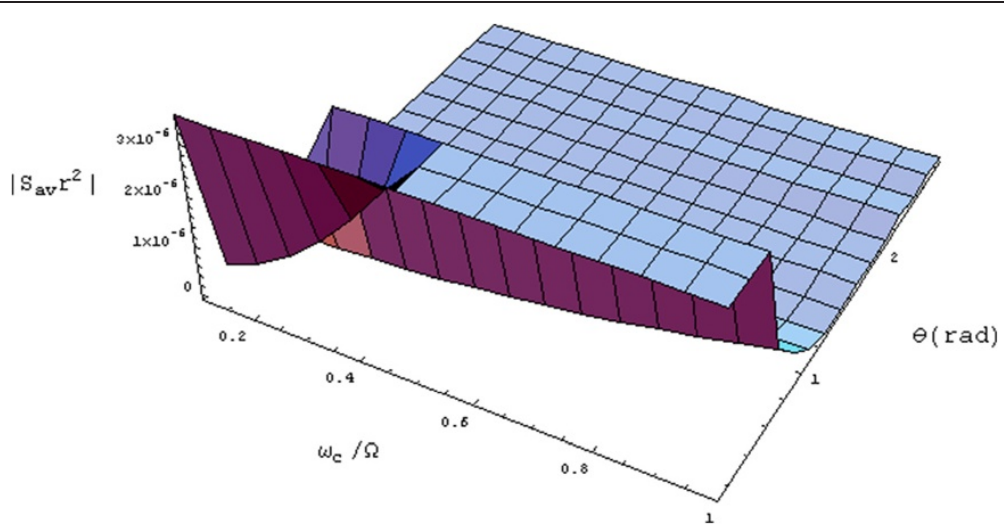

Figure 2 Variation of $\left|r^{2} S_{\mathrm{av}}\right|$ with $\omega_{\mathrm{c}} / \Omega$ at different values of $\boldsymbol{\theta}$ for different parameters. The parameters are as follows: $\omega_{\mathrm{c}} / \Omega=20, r_{\mathrm{c}}=25$ $\mathrm{nm}, \mu=0.01$, inter-nanotube separation $a=30 \mathrm{~nm}, \ell_{c}=500 \mathrm{~nm}, n_{0}=10^{21} \mathrm{~cm}^{-3}, \varphi=1 \mathrm{rad}, \varepsilon_{L}=16, L \times L=2 \times 2 \mathrm{~cm}, e A_{L} / \mathrm{m \omega c}=0.03$.

$$
\begin{aligned}
\overrightarrow{\mathrm{J}}_{2 \Omega} & =-\frac{\pi r_{c}^{2} N 2 n_{0} e \Omega i}{m\left(4 \Omega^{2}-\omega_{p}^{\prime 2} / 2\right)} \overrightarrow{\mathrm{F}}_{p} \\
& =\hat{z} \alpha e^{-\left(x-l_{c}\right)^{2} / r_{0}^{2}} e^{-2 i(\Omega t-\Omega z / c)},
\end{aligned}
$$

where

$$
\alpha=\frac{\pi r_{c}^{2} N n_{0} e \mu}{\left(4 \Omega^{2}-\omega_{p}^{\prime 2} / 2\right)} \frac{\Omega^{2} e^{2} A_{L}^{2}}{m^{2} \omega^{2} c} \frac{\omega \omega_{c}}{\omega^{2}-\omega_{\mathrm{pe}}^{\prime 2} / 2-\omega_{c}^{2}} .
$$

As the base of the carbon nanotube is metallic, there will be an image current density underneath the metal surface,

$$
\overrightarrow{\mathrm{J}}_{2 \Omega}^{\prime}=-\hat{z} \alpha e^{-\left(x+\ell_{c}\right)^{2} / r_{0}^{2}} e^{-2 i(\Omega t-\Omega z / c)}
$$

Let the $y$ and $z$ dimensions of the array be $L \times L$.

The vector potential at a far point due to the current distributions is

$$
\begin{aligned}
\overrightarrow{\mathrm{A}}(\overrightarrow{\mathrm{r}}, t)= & \frac{\mu_{0}}{4 \pi}\left[\int\left\{\overrightarrow{\mathrm{J}}_{2 \Omega}\left(\overrightarrow{\mathrm{r}}^{\prime}, t-R / c\right) / R\right)\right\} d V^{\prime} \\
& \left.\left.+\int\left\{\overrightarrow{\mathrm{J}}_{2 \Omega}^{\prime}\left(\overrightarrow{\mathrm{r}}^{\prime}, t-R / c\right) / R\right)\right\} d V^{\prime}\right],
\end{aligned}
$$

where $R=\left|\overrightarrow{\mathrm{r}}-\overrightarrow{\mathrm{r}}^{\prime}\right| \approx r\left(1-\overrightarrow{\mathrm{r}} \cdot \overrightarrow{\mathrm{r}}^{\prime} / r^{2}\right)=r-\sin \theta \cos \phi x^{\prime}-$ $\sin \theta \sin \phi y^{\prime}-\cos \theta z ; ; r, \theta$, and $\phi$ are the spherical polar coordinates of the point of observation, and we shall assume $\Omega \ell_{\mathrm{c}} / c, \Omega r_{\mathrm{c}} / c<<1$ The above Equation 15 can be written as

$$
\overrightarrow{\mathrm{A}}=\hat{z} \frac{\alpha \mu_{0}}{4 \pi r} e^{-2 i(\Omega t-\Omega r / c)}\left(I_{1}-I_{2}\right)
$$

where

$$
I_{1}=\int_{-1 / 2}^{1 / 2} \int_{-1 / 2}^{1 / 2} \int_{-\infty}^{\infty} e^{-\left(x^{\prime}-\ell_{c}\right)^{2} / r_{0}^{2}} e^{i \psi} d x^{\prime} d y^{\prime} d z^{\prime}
$$

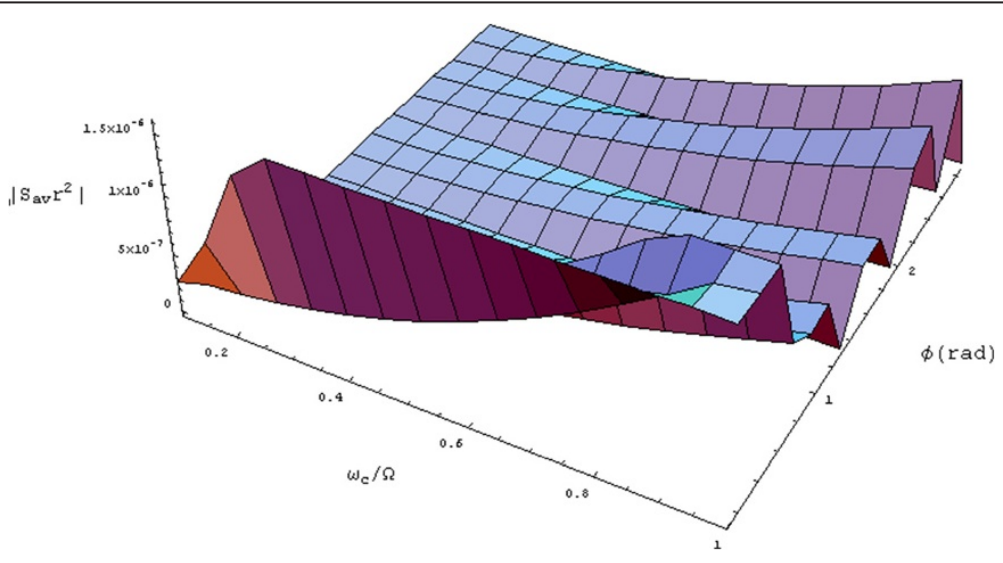

Figure 3 Variation of $\left|r^{2} S_{a v}\right|$ with $\omega_{c} / \Omega$ at different values of $\varphi$ for $\theta=1$ rad. Other parameters are similar to those of Figure 2. 


$$
\begin{aligned}
& I_{2}=\int_{-1 / 2}^{1 / 2} \int_{-1 / 2}^{1 / 2} \int_{-\infty}^{\infty} e^{-\left(x^{\prime}+\ell_{c}\right)^{2} / r_{0}^{2}} e^{i \psi} d x^{\prime} d y^{\prime} d z^{\prime} \\
& \psi^{\prime}=2 \frac{\Omega}{c}\left[z^{\prime}(1-\cos \theta)+x^{\prime} \sin \theta \cos \phi+y^{\prime} \sin \theta \sin \phi\right] .
\end{aligned}
$$

On carrying out integration, $I_{1}$ and $I_{2}$ reduce to

$$
\begin{aligned}
I_{1}= & \frac{\sin [(\Omega L / c)(1-\cos \theta)]}{i(\Omega / c)(1-\cos \theta) \sin \theta \sin \phi} \\
& \sin [(\Omega L / \mathrm{c}) \sin \theta \sin \phi] \sqrt{\pi} \mathrm{r}_{\mathrm{c}} \mathrm{e}^{2 \mathrm{i} \Omega \ell_{\mathrm{c}} / \mathrm{c}}
\end{aligned}
$$

and

$$
\begin{aligned}
I_{2}= & \frac{\sin [(\Omega L / c)(1-\cos \theta)]}{i(\Omega / c)(1-\cos \theta) \sin \theta \sin \phi} \\
& \sin [(\Omega L / \mathrm{c}) \sin \theta \sin \phi] \sqrt{\pi} \mathrm{r}_{\mathrm{c}} \mathrm{e}^{-2 \mathrm{i} \Omega \ell_{\mathrm{c}} / \mathrm{c}},
\end{aligned}
$$

respectively.

From Equations 17 and 18, we obtain

$$
\begin{gathered}
I_{1}-I_{2} \approx \sqrt{\pi} r_{c} \cos \left(\frac{\Omega \ell_{c}}{c}\right) \sin [(\Omega L / c)(1-\cos \theta)] . \\
\frac{\sin [(\Omega L / c) \sin \theta \sin \phi]}{(\Omega / c)(1-\cos \theta) \sin \theta \sin \phi} .
\end{gathered}
$$

The magnetic field at far distance is

$$
\overrightarrow{\mathrm{B}}=\vec{\nabla} \times \overrightarrow{\mathrm{A}} \approx i 2(\Omega / c) \hat{r} \times \overrightarrow{\mathrm{A}} .
$$

The time-average Poynting vector using Equation 20 can be written as

$$
\begin{aligned}
\overrightarrow{\mathrm{S}}_{\mathrm{av}}= & \frac{|B|^{2}}{2 \mu_{0} c} \hat{r} W / \mathrm{m}^{2} \\
= & \hat{r} \frac{r_{c}^{4} \alpha^{2} \mu_{0}^{2} \mu^{2}}{r^{2}}\left(\frac{4 \Omega \ell_{c}}{c}\right)^{2} \sin ^{2}[(\Omega L / c)(1-\cos \theta)] \\
& \sin ^{2}\left[\frac{(\Omega L / \mathrm{c})(\sin \theta \sin \phi]}{(1-\cos \theta)^{2} \sin ^{2} \theta \sin ^{2} \phi} \mathrm{W} / \mathrm{m}^{2} .\right.
\end{aligned}
$$

In Figure 2, we have shown the variation of $\left|r^{2} S_{\mathrm{av}}\right|$ with $\omega_{\mathrm{c}} / \Omega$ at different values of $\theta$ for the following set of parameters: $\omega_{\mathrm{c}} / \Omega=20$, nanotube radius $r_{\mathrm{c}}=25 \mathrm{~nm}, \mu=$ 0.01 , inter-nanotube separation $a=30 \mathrm{~nm}$, length of nanotube $\ell_{\mathrm{c}}=500 \mathrm{~nm}[20], n_{0}=10^{21} \mathrm{~cm}^{-3}, \phi=1 \mathrm{rad}, \varepsilon_{\mathrm{L}}=$ $16, L \times L=2 \times 2 \mathrm{~cm}$, and $e A_{\mathrm{L}} / m \omega c=0.03$. The efficiency of the generated $\mathrm{THz}$ power shows a maximum near $\theta=1$ rad. The efficiency increases with magnetic field strength, and for the above-mentioned parameters, $\omega_{\mathrm{C}} / \Omega=0.1$ corresponds to a magnetic field strength of $100 \mathrm{kG}$. The above parameter of $e A_{\mathrm{L}} / m \omega c=0.03$ can be realized using a $\mathrm{Nd}$ :
YAG laser of $1.06 \mu \mathrm{m}$ with an intensity of $10^{15} \mathrm{~W} / \mathrm{cm}^{2}$. In Figure 3, we have shown the variation of $\left|r^{2} S_{\text {av }}\right|$ with $\omega_{\mathrm{c}} / \Omega$ at different values of $\phi$ for $\theta=1 \mathrm{rad}$. The other parameters are similar to those of Figure 2. The power radiated shows oscillatory behavior with $\phi$.

\section{Conclusions}

An array of carbon nanotubes mounted on a metallic base can be employed to generate $\mathrm{THz}$ radiations. The radiations' pattern is directional with a peak value of power radiated at around $5 \mu \mathrm{W}$, which is quite reasonable. Wang et al. [16] have predicted a maximum efficiency of $5 \mu \mathrm{W}$ for $\mathrm{THz}$ radiations from the armchair carbon nanotube dipole antenna. On comparing with $\mathrm{THz}$ radiation generation from a GaP crystal via the difference frequency generation scheme [3], the efficiency is quite good. One can exploit the resonance condition $\Omega \cong \omega_{p} / \sqrt{8 \varepsilon_{L}}$ for enhancement of efficiency of $\mathrm{THz}$ radiation generation. The application of magnetic field reduces the requirement on the resonance condition. The efficiency of $\mathrm{THz}$ radiation increases with the strength of the applied magnetic field. The analysis is limited to a laser of moderate powers only.

\section{Competing interests}

The authors declare that they have no competing interests.

\section{Authors' contributions}

SJ did the numerical analysis part of the work. JP did the analytical part of the work. RK conceived the idea and helped in designing and drafting the manuscript. All authors read and approved the final manuscript.

\section{Author's information}

SJ did his masters degree in Physics from Barkatullah University, Bhopal (M. P.), India, and at present, he is working towards his Ph.D. degree in Physics. His research interests are in the area of interaction of electromagnetic and electrostatic waves with nanoparticles and nanotubes.

JP is presently the head of the Department of Applied Physics at Samrat Ashok Technological Institute. He did his masters in Physics in 1991 and Ph. D. in 1994 from Barkatullah University, Bhopal (M.P.), India. He worked at I.I.T. Delhi, University of Durban, S.A. and National Central University, Taiwan. His research interests include nonlinear effects in laser matter interaction, plasma physics, and free electron lasers.

RK is currently working as an associate professor at Maulana Azad National Institute of Technology, Bhopal (M.P.), India. He has obtained his masters degree in 1991 from Barkatullah University, Bhopal (M.P.), India and doctoral degree from the University of Leeds (UK) in 1998. In 2011, he has received the Royal Academy of Engineering, London, UK, Research Exchange Award for collaborative research with the University of Bath, UK and also received the UK-India Education Research Initiative (UKIERI phase-II) Award under the Thematic Partnership from British Council Delhi for collaborative research with the University of Bath, UK. His current research interests are ferrite nanoparticles and graphene nanoribbons.

\section{Acknowledgement}

This work was supported by the University Grants Commission, New Delhi, India under the research award scheme.

\section{Author details}

${ }^{1}$ Department of Applied Physics, Samrat Ashok Technological Institute, Vidisha, Madhya Pradesh 464001, India. ²Department of Physics, Maulana Azad National Institute of Technology, Bhopal, Madhya Pradesh 462051, India. 


\section{References}

1. Chan, WL, Deibel, J, Mittleman, DM: Imaging with terahertz radiation. Rep. Prog. Phys. 70, 1325-1379 (2007). doi:10.1088/0034-4885/70/8/R02

2. Bhasin, L, Tripathi, VK: Resonant terahertz generation by optical rectification of an amplitude modulated surface plasma wave. IEEE J. Quant. Electron. 46, 965-969 (2010). doi:10.1109/JQE.2010.2041898

3. Ding, YJ: High-power tunable terahertz sources based on parametric processes and applications. IEEE J. Sel. Top. Quantum Electron. 13, 705-720 (2007)

4. Suizu, K, Koketsu, K, Shibuya, T, Tsutsui, T, Akiba, T, Kawase, K: Extremely frequency-widened terahertz wave generation using Cherenkov-type radiation. Opt. Express 17, 6676-6681 (2009)

5. Bhasin, L, Tripathi, VK: Terahertz generation via optical rectification of $x$-mode laser in a rippled density magnetized plasma. Phys. Plasmas 16, 103105 (2009). doi:10.1063/1.3248303

6. Khoury, J, Saeed, BH, Buchwald, W, Woods, C, Wentzell, S, Krejca, B, Kierstead, J: Electrically tunable surface plasmon source for terahertz applications. IEEE J Selected Topics Quant. Electron. 17, 138-145 (2011). doi:10.1109/JSTQE.2010.2049255

7. Wynw, K, Carey, JJ: An integrated description of terahertz generation through optical rectification, charge transfer, and current surge. Optics Communications 256, 400-413 (2005). doi:10.1016/j.opticom.2005.06.065

8. Son, JH: Terahertz electromagnetic interactions with biological matter and their applications. J. Appl. Phys. 105, 10203 (2009). doi:10.1063/1.3116140

9. Hoffman, MC, Fülöp, A: Intense ultrashort terahertz pulses: generation and applications. J. Phys. D: Appl. Phys. 44, 083001 (2011). doi:10.1088/09534075/44/8/083001

10. Welsh, GH, Wyne, K: Generation of ultrafast terahertz radiation pulses on metallic nanostructured surfaces. Opt. Express 17, 2470-2480 (2009)

11. Garwe, F, Schmidt, A, Zieger, G, May, T, Wyne, K, Hübner, U, Zeisberger, M, Paa, W, Stafast, H, Meyer, HG: Bi-directional terahertz emission from goldcoated nanoratings by excitation via femtosecond laser pulses. Appl. Phys. B 102, 551-554 (2011). doi:10.1007/00340-011-4377-7

12. Bahk, YM, Park, HR, Ahn, KJ, Kim, HS, Ahn, YH, Kim, DS, Abad, JB, Moreno, LM, Vidal, FJG: Anomalous band formation in arrays of terahertz nanoresonators. Phys. Rev. Lett. 106, 013902 (2011). doi:10.1103/PhysRevLett. 106.013902

13. Nemilentsau, AM, Slepyan, GV, Maksimenko, SA: Thermal radiation from carbon nanotubes in the terahertz range. Phys. Rev. Lett. 99, 147403 (2007). doi:10.1103/PhysRevLett. 99.147403

14. Batrakov, KG, Kibis, OV, Kuzhir, PP, de Costa, MR, Portnoi, ME: Terahertz process in carbon nanotubes. J. Nanophotonics 4, 041665 (2010). doi:10.1117/1.3436585

15. Portnoi, ME: Kibis, OV, deCosta. MR: Terahertz applications of carbon nanotubes. Superlattices and Microstructures 43, 399-407 (2008). doi:10.1016/j/spmi.2007.07.026

16. Wang, Y, Wy, Q, He, X, Zhang, S, Zhuang, L: Terahertz radiation from armchair carbon nanotube dipole antenna. Chin. Phys. B 18, 1801-1806 (2009)

17. Wang, $Y, W u, Q$ : Properties of terahertz wave generated by the metallic carbon nanotube antenna. Chin. Opt. Lett. 8, 770-772 (2008)

18. Ramakrishnan, G, Chakkittakandy, R, Paul, CMP: Terahertz generation from graphite. Opt. Exp. 17, 16092-16098 (2009)

19. Dragoman, D, Dragoman, M: Terahertz oscillations in semiconducting carbon nanotube resonant tunneling diodes. Physica E 24, 282-289 (2004)

20. Wang, Y, Kempa, K, Kimball, B, Carlson, JP, Benham, G, Li, WZ, Kempa, T: Rybczynski, Herczynski A. Ren ZF: Receiving and transmitting light like radiowaves: antenna effect in arrays of aligned carbon nanotubes. Appl. Phys. Lett. 85, 2607-2609 (2004)

21. Dagher, M, Chamanara, N, Sounas, D, Martel, R, Caloz, C: Theoretical investigation of traveling wave amplification in metallic carbon nanotubes biased by a dc field. IEEE Trans. Nanotech. 11, $463-471$ (2012). doi:10.1109/TNANO.2011.2175005

22. Parashar, J, Sharma, H: Optical rectification in a carbon nanotube array and terahertz radiation generation. Physica E 44, 2069-2071 (2012). doi:10.1016/j. physe.2012.06.013

doi:10.1186/2228-5326-3-1

Cite this article as: Jain et al: Effect of magnetic field on terahertz generation via laser interaction with a carbon nanotube array. International Nano Letters 2013 3:1.

\section{Submit your manuscript to a SpringerOpen ${ }^{\odot}$ journal and benefit from:}

- Convenient online submission

- Rigorous peer review

- Immediate publication on acceptance

- Open access: articles freely available online

- High visibility within the field

- Retaining the copyright to your article

Submit your next manuscript at $>$ springeropen.com 